\title{
Three-dimensional digital subtraction angiography vs two- dimensional digital subtraction angiography for detection of ruptured intracranial aneurysms: a study of 86 aneurysms
}

\author{
M. Kawashima, T. Kitahara', K. Soma', K. Fujii \\ Departments of Neurosurgery and ${ }^{1}$ Emergency and Critical Care Medicine, Kitasato University, School of Medicine, Kanagawa Japan
}

\begin{abstract}
Aims: Three-dimensional reconstruction of intracranial vessels is of interest for evaluation of aneurysms. This study determined diagnostic difference of three-dimensional digital subtraction angiography (3D-DSA, volume-rendering image) versus 2D-DSA for evaluating ruptured intracranial aneurysms, particularly focusing on the size of aneurysms as depicted in both images. Settings and Design: Sixtynine patients underwent 3D-DSA and 2D-DSA. The relative size of an aneurysm, which is the ratio of the maximal diameter of an aneurysm to the diameter of a major vessel, was compared between imaging techniques. In addition, relative sizes of smaller aneurysms $(<5 \mathrm{~mm})$ were compared with those of larger aneurysms $(>10 \mathrm{~mm})$. Statistical analysis used: For comparison of aneurysm size and location of aneurysm, statistical analysis was performed with the Yates chi square test; statistical significance was set with a $P$ value of less than 0.05 . Results: Sixty-three $(73.3 \%)$ of the 86 total aneurysms were bigger when measured with 3D-DSA versus 2D-DSA. When measured with 3D-DSA, 28 (84.8\%) of the 33 smaller aneurysms were bigger, and $50 \%$ of the larger aneurysms were bigger versus measurements of 2DDSA images $(P<0.05)$. In ACA and ICA territories, which tended to have smaller mean aneurysmal size, relative size of the aneurysm was bigger when measured with 3D-DSA ( $81.5 \%$ and $81.0 \%$, respectively). In MCA, where the mean aneurysmal size was the largest, relative size of aneurysms was bigger when measured with 3D-DSA in 15 cases $(53.6 \%$, $P<0.05)$. In the posterior circulation, aneurysm size was similar between 3D-DSA and 2D-DSA measurements. Conclusions: 3D-DSA, especially volume-rendering images, tends to depict ruptured intracranial aneurysms bigger than 2DDSA. This is particularly true with cerebral aneurysms that are $<5 \mathrm{~mm}$ in size and are located in the anterior circulation, especially ICA and ACA territories.
\end{abstract}

Key-words: Cerebral aneurysm, subarachnoid hemorrhage, three-dimensional digital subtraction angiography, two-dimensional digital subtraction angiography.

\section{Introduction}

Three-dimensional (3D) reconstruction of intracranial vessels at imaging is of particular interest for the evaluation of aneurysms. Precise visualization of the aneurysm and its relationship with parent vessels are fundamental factors that must be assessed before treatment. Knowledge of the size of an aneurysm is essential before endovascular and neurosurgical procedures are performed. This information can be obtained, in part, using 3D computed tomographic angiography ${ }^{[1-5]}$ and magnetic resonance angiography. ${ }^{[6-8]}$ Compared with digital subtraction angiography (DSA), however, these techniques have inferior spatial resolution and lower sensitivity to detect small $(<3 \mathrm{~mm})$ intracranial aneurysms. ${ }^{[6,7,9,10,11]}$ The current standard of reference in the diagnostic workup of intracranial aneurysms is DSA, which can depict aneurysms with a diameter $<3$ $\mathrm{mm} \cdot{ }^{[12]}$ Recently, 3D-DSA has been used to detect intracranial aneurysms in cases of acute subarachnoid hemorrhage. Some authors have reported that 3D-DSA and DSA together can depict more intracranial aneurysms than DSA alone. ${ }^{[13,14,15]}$ The objective of this study was to evaluate the diagnostic benefit of 3D-DSA compared with DSA in detecting intracranial aneurysms, focusing especially on the relative size of the aneurysm depicted by each imaging technique.

\section{Subjects and methods}

A total of 124 consecutive patients with ruptured intracranial aneurysms were transferred to our hospital between January 2003 and May 2004. Of them, the 69 patients who underwent 3D-DSA were enrolled in this study. The study population included 47 women and 22 men ranging in age from 29 
to 81 years (mean, 63.5 years). All patients underwent 3DDSA within 3 days from the initial onset. Informed consents were obtained before the procedure. All angiographic procedures were performed using a DSA unit (Advance LCN plus, GE Medical Systems). 3D-DSA images were produced by a workstation (Advantage Workstation 4.0, GE Medical Systems) using data from rotational angiographies. Rotational angiography was performed with a $203.6^{\circ}$ rotation of the $\mathrm{C}$ arm in 5.8 seconds. The matrix size of each frame was $512 \mathrm{x}$ 512 pixels. Seventeen to $23 \mathrm{ml}$ of contrast medium (Iohexol, $300 \mathrm{mg} / \mathrm{dl}$ ) was injected at a rate of 3 (internal carotid and vertebral arteries) to $4 \mathrm{ml} / \mathrm{s}$ (common carotid artery) via a catheter positioned at the cervical portion of the carotid arteries and/or vertebral arteries. Both mask data and contrast data were automatically transferred to the workstation. Threedimensional subtraction images were automatically produced on the workstation by a 3D reconstruction algorithm based on the algebraic reconstruction technique. Reconstructed images, including shaded surface display and volume rendering, were produced from the data. Minimum density threshold processing was automatically performed at the same levels. The maximum diameter of each aneurysm was calculated on the $3 \mathrm{D}$ workstation. In definitely detected aneurysms, we compared the relative size of the aneurysm, which is the ratio of the maximal diameter of an aneurysm to the diameter of a major vessel, between measurements made using 2D-DSA and 3D-DSA. Relative size of the aneurysm in both images was measured in the same direction, which was chosen based on achieving the greatest size estimate. Differences between 2DDSA and 3D-DSA estimates of aneurysm size were compared for different regions. We also compared the 2D-DSA and 3DDSA estimates of relative sizes for smaller $(<5 \mathrm{~mm})$ and larger $(>10 \mathrm{~mm}$ ) aneurysms. For comparison of aneurysm size and location of aneurysm, statistical analysis was performed with the Yates chi square test; statistical significance was set with a $\mathrm{P}$ value of less than 0.05 .

\section{Results}

3D-DSA confirmed 86 aneurysms in 69 patients in the present study. Eleven of 69 patients had multiple aneurysms. The aneurysms were located in the internal carotid artery (ICA, $n=21$ ), middle cerebral artery (MCA, $n=28$ ), anterior cerebral artery ( $\mathrm{ACA}, \mathrm{n}=27$ ), vertebral artery-posterior inferior cerebellar artery (VA-PICA, $n=3)$, distal PICA $(n=1)$, basilar artery-superior cerebellar artery (BA-SCA, $n=3)$, and BA top $(n=3)$. The size of aneurysms was measured using 3D-DSA images. The mean maximal sizes of aneurysms were $6.77,7.65,6.01,6.15$, and $5.67 \mathrm{~mm}$ in ICA, MCA, ACA, VAPICA and distal PICA, and BA-SCA and PICA, respectively. Sixty-three of $86(73.3 \%)$ aneurysms were bigger when measured with 3D-DSA versus 2D-DSA. Agreement between aneurysm size estimates using 2D-DSA and 3D-DSA were compared for smaller $(<5 \mathrm{~mm})$ and larger $(>10 \mathrm{~mm})$ aneurysms.
In 33 smaller aneurysms, 28 (84.8\%) were bigger when measured using 3D-DSA versus 2D-DSA, whereas $50 \%$ of larger aneurysms were bigger when measured using 3D-DSA versus 2D-DSA ( $\mathrm{P}<0.05$, Table 1). Relative size of aneurysms was typically bigger when measured using 3D-DSA versus $2 \mathrm{D}$ DSA, especially in ACA and ICA territories (81.5\% and $81.0 \%$, respectively), which correlated with smaller mean aneurysmal size. In MCA, where the mean aneurysmal size was the largest, relative size of an aneurysm was bigger when measured with 3D-DSA in only 15 cases $(53.6 \%, \mathrm{P}<0.05)$. There was no difference between aneurysm size estimates in the posterior circulation [Table 2].

\section{Discussion}

Several studies have reported that 3D-DSA provides more detailed information than 2D-DSA for evaluating cerebral aneurysms. ${ }^{[13]-15]}$ Some authors have demonstrated that 3D-DSA is valuable for evaluating the potential for endovascular treatment by allowing observation and analysis from multiple directions. ${ }^{[16]-[18]}$ Minor variations in size of the aneurysm will affect management decisions. For the visualization of the aneurysmal neck and its relationship to neighboring arteries, 3D-DSA images were significantly superior to those of 2D-DSA, including. rotational DSA. The size of an aneurysm and the diameter of the neck can be correctly measured using 3D-DSA, as was shown in a recent in vivo study ${ }^{[14]}$. In the present study, we demonstrated differences between aneurysmal size measurements obtained using 3D-DSA and 2D-DSA. 3D-DSA was more sensitive for detection of smaller aneurysms. Relative sizes of aneurysms were compared between imaging techniques because the true size of aneurysms could not be confirmed for the 2DDSA images. In our study, $73 \%$ of aneurysms examined were greater in size when measured using 3D-DSA versus 2D-DSA.

\begin{tabular}{lcc}
\hline $\begin{array}{c}\text { Table 1: Comparison of relative sizes of intracranial aneurysms } \\
\text { between 2D-DSA and 3D-DSA }\end{array}$ \\
\hline \multicolumn{4}{c}{ 3D-DSA > 2D-DSA } & 3D-DSA < 2D-DSA \\
All aneurysms, $n=86$ & $63(73 \%)$ & $23(27 \%)$ \\
Aneurysms $<5 \mathrm{~mm}, n=33$ & $28(85 \%)$ & $5(15 \%)^{*}$ \\
Aneurysms $>10 \mathrm{~mm}, n=14$ & $7(50 \%)$ & $7(50 \%)$
\end{tabular}

${ }^{*} P<0.05$ (Yates chi square test).

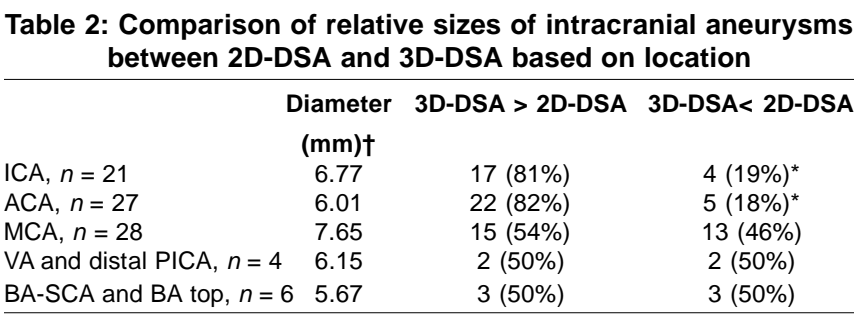

ACA, anterior cerebral artery; BA, basilar artery; ICA, internal carotid artery; MCA, middle cerebral artery; PICA, posterior inferior cerebellar artery; SCA, superior cerebellar artery; VA, vertebral artery.

${ }^{*} P<0.05$ (Yates chi square test).

† Diameter determined using 3D-DSA images. 
In particular, smaller aneurysms $(<5 \mathrm{~mm})$ tended to be greater in size when measured using $3 \mathrm{D}-\mathrm{DSA}(85 \%)$ versus $2 \mathrm{D}-\mathrm{DSA}$. Of larger aneurysms (>10 mm), only $50 \%$ were bigger with 3D-DSA versus 2D-DSA $(\mathrm{P}<0.05)$. In addition, aneurysms located in ACA and ICA territories were larger with 3D-DSA measurements versus aneurysms located in MCA $(\mathrm{P}<0.05)$, PICA, and BA territories $(82 \%$ and $81 \%$ vs. $54 \%, 50 \%$, and $50 \%$, respectively). Average maximal 3D-DSA diameters of aneurysms located in ACA, ICA, and MCA territories were 6.01, 6.77 , and $7.65 \mathrm{~mm}$, respectively. These results suggest that the smaller aneurysms located in the anterior circulation, especially ACA and ICA territories, are more easily detected using 3DDSA than 2D-DSA. One reason why aneurysm size may be underestimated by 2D-DSA is that part of the dome of the aneurysm frequently overlaps with the parent vessel, thus accurate measurements of 2D-DSA images is difficult. Furthermore, the shape of an aneurysm, including its neck and bleb, is depicted more clearly in 3D-DSA than 2D-DSA. Aneurysms located in the posterior circulation were more equally depicted by 3D-DSA and 2D-DSA images, probably because the number of vessels around the aneurysms was rather small and the relationship between the aneurysms and parent vessels was more easily detected than those in the anterior circulation. Although it is not clear if 3D-DSA is the truth regarding the true aneurysm size, 3D-DSA would be helpful to detect a cerebral aneurysm, because it tends to depict intracranial aneurysms bigger than 2D-DSA

In the present study, we used a volume-rendering method for reconstruction. This method has some advantages over maximum intensity projection (MIP) and shaded surface display (SSD) techniques. First, the entire DSA dataset can be incorporated within the 3D image, whereas a small fraction of data is displayed on MIP and SSD images. Second, the number, density, and opacity of voxels can be adjusted separately to allow change in the transparency of selected materials. Finally, the 3D appearance is maintained and enables a good analysis of the relationships among vascular structures.

The advantage of 3D-DSA may have an impact on the patient's therapy, and 3D-DSA may augment the role of 2D-DSA as the criterion standard in assessing intracranial aneurysms. However, it is important to note that image quality is degraded by some artifacts, including equipment vibration, obscuration of small vessels originating near the aneurysm and distal cerebral arteries, and patient movement. Patient movement was not marked in this study because most patients were sedated and intubated to avoid aneurysmal re-rupture immediately after diagnosis of subarachnoid hemorrhage.

3D-DSA, especially volume-rendering images, had greater advantage in depicting ruptured intracranial aneurysms than 2D-DSA. In particular, this advantage was prominent in detecting cerebral aneurysms that were $<5 \mathrm{~mm}$ in size and were located in the anterior circulation, especially in the ICA and ACA territories.

\section{Acknowledgement}

The authors thank Hitoshi Maeda, RT, for technical support with angiographic procedures. The authors thank Becky Norquist for reviewing the manuscript.

\section{References}

1. Alberico RA, Patel M, Casey S, Jacobs B, Maguire W, Decker R. Evaluation of the circle of Willis with three-dimensional CT angiography in patients with suspected intracranial aneurysms. A.JNR Am J Neuroradiol 1995;16:1571-80.

2. Korogi Y, Takahashi M, Katada K, Ogura Y, Hasuo K, Ochi M, et al. Intracranial aneurysms: detection with three-dimensional CT angiography with volume rendering - comparison with conventional angiographic and surgical findings. Radiology 1999;211:497-506.

3. Kawashima M, Matsushima T, Miyazono M, Hirokawa E, Baba H. Two surgical cases of internal carotid-ophthalmic artery aneurysms: special reference to the usefulness of three-dimensional CT angiography. Neurol Res 2002;24:8258.

4. Carvi y Nievas MN, Haas E, Hollerhage HG, Drathen C. Complementary use of computed tomographic angiography in treatment planning for posterior fossa subarachnoid hemorrhage. Neurosurgery 2002;50:1283-89.

5. Kangasniemi M, Makela T, Koskinen S, Porras M, Poussa K, Hernesniemi J Detection of intracranial aneurysms with two-dimensional and three-dimensional multislice helical computed tomographic angiography. Neurosurgery 2004;54:336-41.

6. Ida M, Kurisu Y, Yamashita M. MR angiography of ruptured aneurysms in acute subarachnoid hemorrhage. AJNR Am J Neuroradiol 1997; 18:1025-32.

7. Chung TS, Joo JY, Lee SK, Chien D, Laub G. Evaluation of cerebral aneurysms with high-resolution MR angiography using a section-interpolation technique: correlation with digital subtraction angiography. A.JNR Am .J Neuroradiol $1999 ; 20: 229-35$.

8. Okahara M, Kiyosue H, Yamashita M, Nagatomi H, Hata H, Saginoya T, et al. Diagnostic accuracy of magnetic resonance angiography for cerebral aneurysms in correlation with 3D-digital subtraction angiographic images: a study of 133 aneurysms. Stroke 2002;33:1803-8.

9. Metens T, Rio F, Baleriaux D, Roger T, David P, Rodesch G. Intracranial aneurysms: detection with gadolinium-enhanced dynamic three-dimensional MR angiography-initial results. Radiology 2000;216:39-46.

10. Korogi Y, Takahashi M, Mabuchi N, Nakagawa T, Fujiwara S, Horikawa Y, et $a l$. Intracranial aneurysms: diagnostic accuracy of MR angiography with evaluation of maximum intensity projection and source images. Radiology 1996;199:199-207.

11. Young N, Dorsch NW, Kingston RJ. Pitfalls in the use of spiral CT for identification of intracranial aneurysms. Neuroradiology 1999;41:93-9.

12. Urbach H, Zentner J, Solymosi L. The need for repeat angiography in subarachnoid haemorrhage. Neuroradiology 1998;40:6-10.

13. Kojima A, Yamaguchi N, Okui S. Three dimensional digital subtraction angiography imaging of a ruptured aneurysm on the anterior communicating artery. Surg Neurol 2001; 58:49-53.

14. Hochmuth A, Spetzger U. Comparison of three-dimensional rotational angiography with digital subtraction angiography in the assessment of ruptured cerebral aneurysms. A.JNR Am J Neuroradiol 2002;23:1199-205.

15. Sugahara T, Korogi Y, Nakashima K, Hamatake S, Honda S, Takahashi M. Comparison of $2 \mathrm{D}$ and $3 \mathrm{D}$ digital subtraction angiography in evaluation of intracranial aneurysms. A.JNR Am J Neuroradiol 2002;23:1545-52.

16. Anxionnat R, Bracard S, Ducrocq X, Trousset Y, Launay L, Kerrien E, et al. Intracranial aneurysms: clinical value of $3 \mathrm{D}$ digital subtraction angiography in the therapeutic decision and endovascular treatment. Radiology 2001;218:799808.

17. Abe T, Hirohata M, Tanaka N, Uchiyama Y, Kojima K, Fujimoto K, et al. Clinical benefits of rotational 3D angiography in endovascular treatment of ruptured cerebral aneurysm. A.JNR Am J Neuroradiol 2002;23:686-8.

18. Kiyosue H, Tanoue S, Okahara M, Hori Y, Nakamura T, Nagatomi H, et al. Anatomic features predictive of complete aneurysm ocelusion can be determined with three-dimensional digital subtraction angiography. AJNR Am J Neuroradiol 2002;23:1206-13.

Accepted on 26-08-2005 


\section{Invited Comments}

This study compares the size of aneurysms when measured on standard 2D DSA in comparison with 3D volume rendered DSA. This is important because 3D imaging is increasingly recognized as useful for both aneurysm detection and therapy planning. As a result, the authors found that the aneurysms appear larger when measured on the $3 \mathrm{D}$ images in relation to the surrounding arteries. While it is not clear which of the methods (2D or 3D) shows the 'truth' the study clearly demonstrates the main problem that we still have with 3D imaging. There are no standards for how to use MIP, volume rendering, surface shading, and so on. Thus it is likely that the same study would lead to completely different results when another hardware or workstation for post-processing is used. It is well known that the size of (arterial) structures will vary when thresholds are changed. Increasing the lower threshold will lead to a decrease in size of the arteries and tiny structures will disappear soon while bigger structures like aneurysms will keep their 'size' on volume rendered images even with higher thresholds. This fact may explain the result of this study. Modern workstations provide wonderful tools to create realistic 3D models from CT or MR data in excellent quality. However, as long as 3D imaging still relies on the user-dependent interaction with a workstation there will be no reproducible results. Therefore, the main task for the near future is the definition of standard procedures for 3D imaging to create reproducible results that are independent of workstations and users. As long as this is not guaranteed one must be aware that 3D imaging is not a defined method that has to be evaluated in each single institution.

\section{Bernd F. Tomandl}

Department of Neuroradiology, Bremen East Central Hospital, Züricherstr. 40, D-28325 Bremen, Germany E-mail: bernd_tomandl@hotmail.com 